\title{
Selective chemical oxidation or reduction of chlorophenols with potassium nitrosodisulfonate
}

\author{
Elisa Leyva,* Ismael Crispin, Edgar Moctezuma, and Socorro Leyva \\ Chemistry Department, Universidad Autonoma de San Luis Potosi \\ Manuel Nava \# 6, San Luis Potosi S.L.P. Mexico 78210 \\ E-mail: elisa@uaslp.mx
}

(received 05 Sep 03; accepted 20 Jan 03; published on the web 25 Jan 04)

\begin{abstract}
The selective chemical oxidation or reduction of several substituted chlorophenols (2-chloro, 4chloro, and 2,4-dichloro) was carried out with potassium nitrosodisulfonate. The reaction was followed by NMR and HPLC. This reaction was strongly dependent on the amount of potassium nitrosodisulfonate, the position of the chloro-substituent on the aromatic ring, the $\mathrm{pH}$ and temperature of reaction. At short reaction times, 4-chorophenol was selectively oxidized to 1,4benzoquinone and 2-chlorophenol to 2-chloro-1,4-benzoquinone. In contrast, 2,4-dichlorophenol was selectively reduced to 4-chlorophenol.
\end{abstract}

Keywords: Selective oxidation, selective reduction, chlorophenols, organic contaminants

\section{Introduction}

Chlorophenols represent an important class of environmental water pollutants. ${ }^{1}$ Many of these compounds are present in waste waters from petrochemical, coal tar, plastic and pesticidal chemical industries, which produce them as chemical intermediates or generate them during chlorination of effluents containing phenolic compounds. ${ }^{2-4}$ Industrial waste waters are not the only source of chlorophenols. They could be present in domestic waters since they are widely used as pesticides, desinfectants and antiseptics. In addition, the common water treatment with chlorine could generate chlorophenols since chlorine reacts quite fast with phenols. ${ }^{5}$

Chlorophenols can be oxidized by chemical, photochemical and microbiological processes. ${ }^{2,3}$ However, biological treatment of waste waters containing chlorinated phenols requires longer retention times and in the case of higher concentrations, the activated sludge formation can be substantially intoxicated and suppressed. ${ }^{6}$

The photochemical degradation of a given chlorophenol is strongly dependent on its absorption spectra. ${ }^{7}$ In this type of degradation, a haloaromatic compound reacts with $\bullet \mathrm{OH}$ radical and the halogen atom is replaced by a hydroxyl group in the aromatic ring. The type of products that are formed depends on the position of the chlorine atom. For example, 
2-chlorophenol gives predominantly pyrocatechol, on the other hand, 3-chlorophenol gives resorcinol. Photochemical irradiation of 4-chlorophenol gives several compounds, such as; hydroquinone, benzoquinone and benzenetriol. These compounds are obtained in acidic and basic conditions, but their concentrations change depending on the $\mathrm{pH}$ of the solution. ${ }^{8}$

Chlorophenols can also be oxidized by photocatalytic processes, in which photo-induced holes in semiconductor particles oxidize hydroxide ions or water molecules adsorbed on the surface of the particles to produce $\bullet \mathrm{OH}$ radicals which subsequently attack adsorbed organic molecules. ${ }^{9-17}$ In the photocatalytic oxidation reported by Tseng and Huang, ${ }^{18}$ the complete mineralization of 3-chlorophenol, 4-chlorophenol, 2,4-dichlorophenol, 2,5-dichlorophenol and 2,6-dichlorophenol was observed. Several research groups ${ }^{19,20}$ have reported the reaction mechanisms and the reaction rate equations for the photocatalytic degradation of different chlorophenols. Photocatalytic degradation pathways, product yields and reaction rates depend on the reaction conditions. In the case of phenol oxidation, Okamoto suggested that phenol undergoes complete mineralization by three different pathways and identified hydroquinone, catechol, 1,2,4-benzenetriol, 2-hydroxybenzoquinone and benzoquinone in the degradation pathways. ${ }^{21,22}$ In two recent papers, Jenks et al. presented the photocatalytic oxidation of 4chlorophenol with $\mathrm{TiO}_{2}{ }^{23,24} \mathrm{~A}$ myriad of intermediate compounds were identified and characterized and a cascade of degradation pathways was presented. In the photocatalytic degradation of 4-chlorophenol the two primary oxidation products are hydroquinone and 4chlorocatechol. Then, hydroquinone is almost quantitatively oxidized into 1,2,4-benzenetriol. Both intermediates, hydroquinone and 4-chlorocatechol, are eventually decomposed into water, carbon dioxide and chlorine ions.

There are many reports on the chemical oxidation of organic compounds by means of chemical oxidants. ${ }^{25,26}$ In these reactions two types of chemical oxidation could occur. In a total oxidation or mineralization, organic compounds are oxidized all the way to water and carbon dioxide. In a partial oxidation, an organic compound is transformed into another compound or a mixture of compounds. Hydrogen peroxide is a strong oxidant that is frequently used in the oxidation of organic pollutants present in water. It is easy to handle, miscible with water and cheap. Its high reactivity is mostly due to the weak $\mathrm{O}-\mathrm{O}$ bond, that generates $\bullet \mathrm{OH}$ radicals upon cleavage. This reaction is facilitated with the presence of metal oxides. In fact, Barbeni et al. investigated the chemical oxidation of 2-chlorophenol, 3-chlorophenol, 3,4-dichlorophenol, and 2,4,5-trichlorophenol by means of the Fenton Reagent $\left(\mathrm{Fe}^{2+}+\mathrm{H}_{2} \mathrm{O}_{2}\right)$ in the presence of oxygen. ${ }^{27}$ Ozone also has been used to oxidize organic contaminants and it is stronger than hydrogen peroxide. ${ }^{28}$ It is usually generated in situ, it can react directly with the organic contaminant since it is a strongly dipolar molecule or it can react in an indirect manner by means of the $\bullet \mathrm{OH}$ radicals that are produced upon its decomposition. In any case, the mechanistic pathway observed in this oxidation depends on the $\mathrm{pH}$ of the solution.

One of the major trends in modern organic chemistry is the development or finding of very selective reagents. In the area of oxidation reactions of organic compounds the number of such selective oxidizing agents is still small. One of the few of these agents is potassium 
nitrosodisulfonate or Fremy's radical. This salt has been reported to selectively oxidize phenol to the corresponding quinone. ${ }^{29}$ In a short communication, two of us reported the total oxidation of chlorophenols with strong oxidants. ${ }^{30}$ In the present paper, the selective chemical oxidation or reduction of several substituted chlorophenols (4-chloro, 2,4-dichloro and 2-chlorophenol) with potassium nitrosodisulfonate and the mechanism of the oxidation are presented.

\section{Experimental Section}

General Procedures. NMR spectra were obtained on a Bruker AC-200 spectrometer. Solutions of potassium nitrosodisulfonate and each chlorophenol $(10 \mathrm{mg} / \mathrm{mL})$ in deuterated water were prepared for ${ }^{1} \mathrm{H}$ NMR studies using TMS as an external standard. Solutions of potassium nitrosodisulfonate and each chlorophenol $(20 \mathrm{mg} / \mathrm{mL})$ in deuterated water were prepared for ${ }^{13} \mathrm{C}$ NMR studies using deuterated acetone as an external reference. The reactions were performed in $5 \mathrm{~mm}$ NMR tubes for ${ }^{1} \mathrm{H}$ NMR and in $10 \mathrm{~mm}$ NMR tubes for ${ }^{13} \mathrm{C}$ NMR. The reaction tubes were introduced in the magnet and kept inside during the reaction. ${ }^{1} \mathrm{H}$ and ${ }^{13} \mathrm{C}$ spectra were obtained at several reaction times. Chemical shifts were recorded in ppm. Each of the reaction samples was analyzed by HPLC using a Waters Chromatograph model 600E equipped with a UV detector Waters 490 tuned at $280 \mathrm{~nm}$ and a NovaPak Phenyl column (60 A, $4 \mu \mathrm{m}, 3.9 \times 100 \mathrm{~mm}$ ). The mobile phase was $11 \mathrm{mM}$ citric acid $/ 0.055 \mathrm{mM}$ EDTA $/ 45 \%$ methanol and the flow rate was 0.8 $\mathrm{mL} / \mathrm{min}$. The concentration of the starting material and several intermediates was determined by HPLC. The identification of intermediates by HPLC was performed comparing the retention times and the UV spectra with those of authentic samples.

Table 1. Retention times for chlorophenols and reaction products by HPLC

\begin{tabular}{ll}
\hline Compound & Retention Time (min) \\
\hline 1,4-benzoquinone & 1.90 \\
phenol & 2.43 \\
2-chloro-1,4-benzoquinone & 3.13 \\
2-chlorophenol & 4.18 \\
4-chlorophenol & 5.56 \\
2,4-dichorophenol & 11.33 \\
\hline
\end{tabular}

Some of the samples were analyzed by GC with MS detector in order to identify the reaction intermediate products. The organic compounds in the reaction samples were extracted with ethyl acetate and concentrated in a rotary evaporator. Then, this solution was analyzed in a Hewlett Packard gas chromatograph Model 5890 series II equipped with a quadrupole mass detector Hewlett Packard model 5972. The chromatographic separation was performed by a capillary column (crosslinked 5\% Me-Phenyl-Silicone, $0.2 \mathrm{~mm}$ i.d. 0.33 x $25 \mathrm{~m}$ ) using Helium as carrier gas. 
Materials. Potassium nitrosodisulfonate was prepared according to the literature procedure. ${ }^{29}$ Due to its radical character, it is highly reactive and unstable and it has been reported to decompose and explode. The organic compounds used were 2-chlorophenol, 4-chlorophenol and 2,4-dichlorophenol (99.9\% purity, Aldrich). All solutions were prepared with deionized water. Mobile phase for HPLC analysis was prepared with methanol (HPLC grade, Fisher Scientific), citric acid (99.9\% purity Aldrich) and EDTA (Mallinckrodt).

General oxidation procedure. Solutions of each chlorophenol $(100 \mathrm{mg} / \mathrm{mL})$ in deionized water were prepared. An aqueous solution of the oxidant was added keeping the relation 2 to 4 moles of the oxidant per mol of chlorophenol. Unless otherwise stated, the reaction mixture was kept at room temperature with stirring for 8 hours and samples were taken every 30 minutes. The reaction mixtures were analyzed by HPLC, NMR and GC-MS.

\section{Results and Discussion}

A set of standard conditions was established for degradation of the several chlorophenols (2chloro, 4-chloro and 2,4-dichloro). The concentration of starting materials and various intermediates was determined by HPLC analysis with a UV detector. The retention times of starting chlorophenols and reaction products are given in Table 1 in the experimental section.

Solutions of chlorophenols with potassium nitrosodisulfonate were allowed to react for several hours and the mixtures were analyzed every 30 minutes. Upon HPLC analysis, the parent chlorophenol peak was observed to decrease while the presence of new peaks, corresponding to products of reaction, were detected. The reaction of the several chlorophenols with $\bullet \mathrm{ON}\left(\mathrm{SO}_{3} \mathrm{~K}\right)_{2}$ indicated the presence of several oxidation or reduction products such as phenol, 1,4benzoquinone and 2-chloro-1,4-benzoquinone (Figure 1).
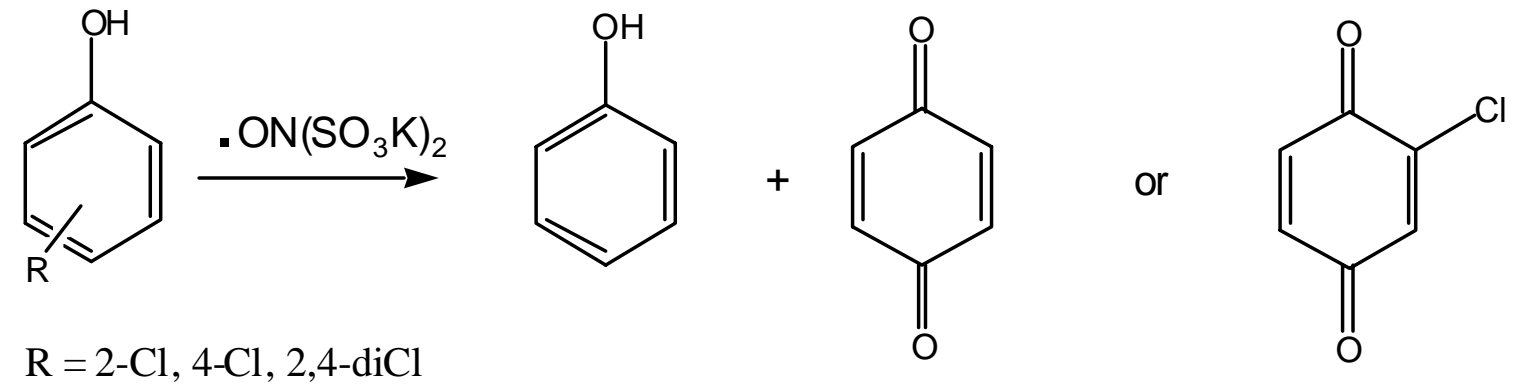

Figure 1. Selective chemical oxidation or reduction of chlorophenols with potassium nitrosodisulfonate.

The effect of the amount of potassium nitrosodisulfonate was investigated (Table 2). As it is expected, an enhancement in the percent of chlorophenol degradation was observed upon increasing the amount of potassium nitrosodisulfonate from 2 to 4 moles. It is interesting to 
realize that even with 4 moles of this reagent the degradation of 2-chlorophenol was only $58 \%$. This clearly indicates that the reactivity of a given chlorophenol depends on the position of the chloro-substituent in the aromatic ring.

Table 2. Degradation of chlorophenol with potassium nitrosodisulfonate as a function of the amount of oxidant

\begin{tabular}{lcc}
\hline Compound & \% Degradation & $\begin{array}{c}\text { Mol of potassium } \\
\text { nitrosodisulfonate/mol of chlorophenol }\end{array}$ \\
\hline 2-chlorophenol & 33 & 2 \\
4-chlorophenol & 52 & 2 \\
2,4-dichlorophenol & 71 & 2 \\
2-chlorophenol & 58 & 4 \\
4-chlorophenol & 83 & 4 \\
2,4-dichorophenol & 73 & 4 \\
\hline
\end{tabular}

The effect of reaction temperature on the percent of degradation was also investigated. For a set of reactions, the aqueous solution of the chlorophenol $(1 \mathrm{~mol})$ and potassium nitrosodisulfonate (4 moles) was refluxed for several hours and monitored every 30 minutes. Under these conditions, total degradation was observed for 4-chlorophenol and 2,4dichlorophenol. However, 2-chlorophenol gave only $89 \%$ degradation (Table 3).

Table 3. Degradation of chlorophenol as a function of temperature

\begin{tabular}{lcc}
\hline Compound & \% Degradation & Temperature ${ }^{\circ} \mathrm{C}$ \\
\hline 2-chlorophenol & 58 & 25 \\
4-chlorophenol & 83 & 25 \\
2,4-dichlorophenol & 73 & 25 \\
2-chlorophenol & 89 & 94 \\
4-chlorophenol & 100 & 94 \\
2,4-dichlorophenol & 99 & 94 \\
\hline
\end{tabular}

The effect of $\mathrm{pH}$ on the degradation of a given chlorophenol $(1 \mathrm{~mol})$ with potassium nitrosodisulfonate (4 moles) was also investigated (Table 4) Three sets of experiments were monitored, a series of solutions with the natural $\mathrm{pH}$ of the solution and a series of solutions with $\mathrm{pH} 3$ or 9. The $\mathrm{pH}$ was modified upon addition of sulfuric acid or sodium hydroxide. A dramatic increase in the degradation was observed when the $\mathrm{pH}$ was raised to 9 (Figure 2). In fact, an enhancement on the reactivity has been observed with other oxidants when the oxidation is under basic conditions. ${ }^{31}$ Since this reaction is thought to proceed through a radical mechanism these conditions favor the formation of several radical intermediates in the reaction mixture. 
Table 4. Degradation of chlorophenol as a function of initial $\mathrm{pH}$

\begin{tabular}{lcc}
\hline Compound & \% Degradation & $\mathrm{pH}$ \\
\hline 2-chlorophenol & 30 & 3 \\
4-chlorophenol & 50 & 3 \\
2,4-dichlorophenol & 56 & 3 \\
2-chlorophenol & 58 & 5.5 \\
4-chlorophenol & 83 & 5.3 \\
2,4-dichlorophenol & 73 & 5.8 \\
2-chlorophenol & 82 & 9 \\
4-chlorophenol & 86 & 9 \\
2,4-dichlorophenol & 85 & 9 \\
\hline
\end{tabular}

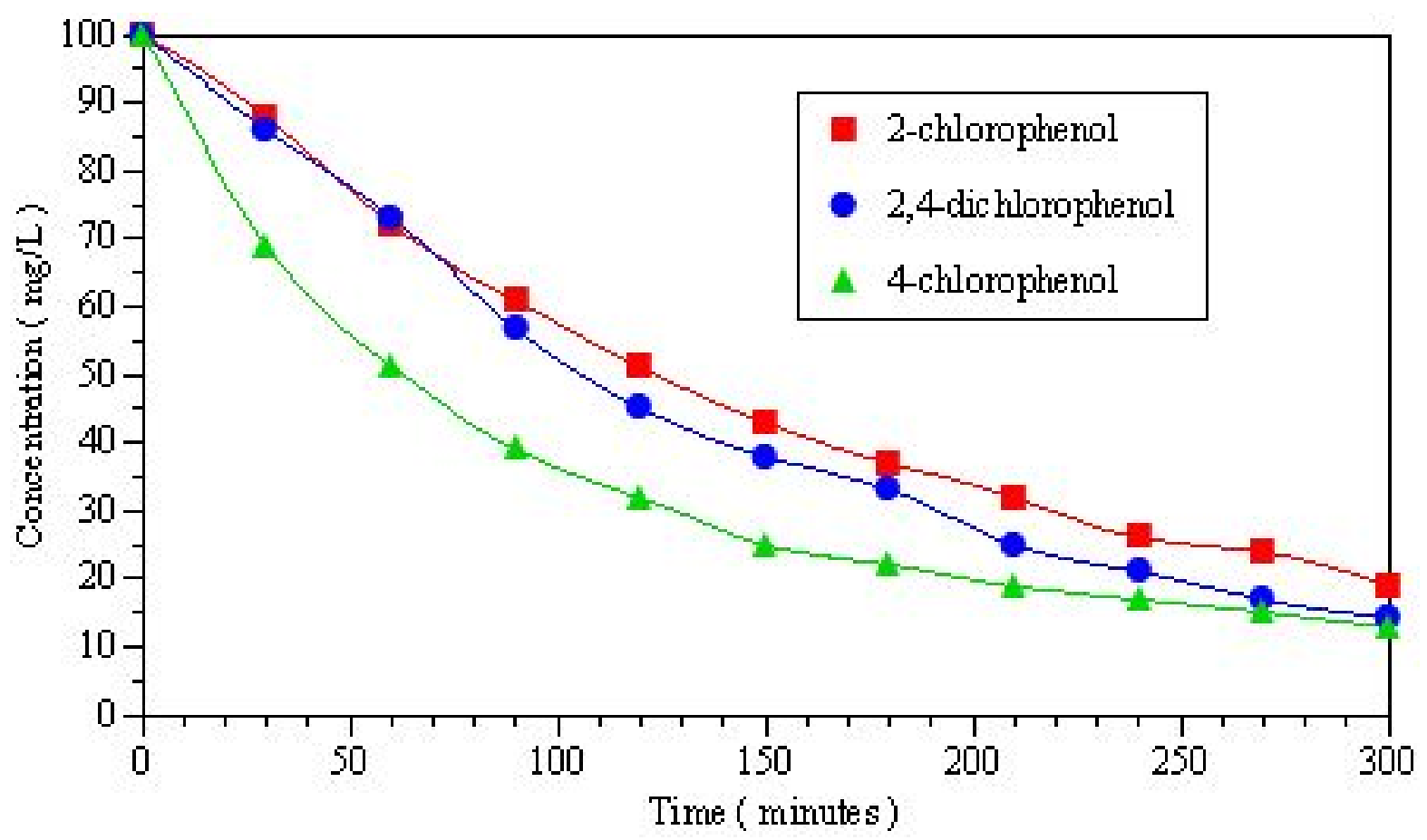

Figure 2. Degradation of a given chlorophenol (1 mol) with potassium nitrosodisulfonate (4 moles) at room temperature and $\mathrm{pH} 9$.

In the reaction of 2-chlorophenol (Figure 3) with potassium nitrosodisulfonate the formation of several intermediates such as phenol and 2-chloro-1,4-benzoquinone was observed. In this case, both intermediates were obtained in quite moderate yields. These product intermediates accumulate in the first five hours of reaction and eventually started to degradate at longer reaction times. 


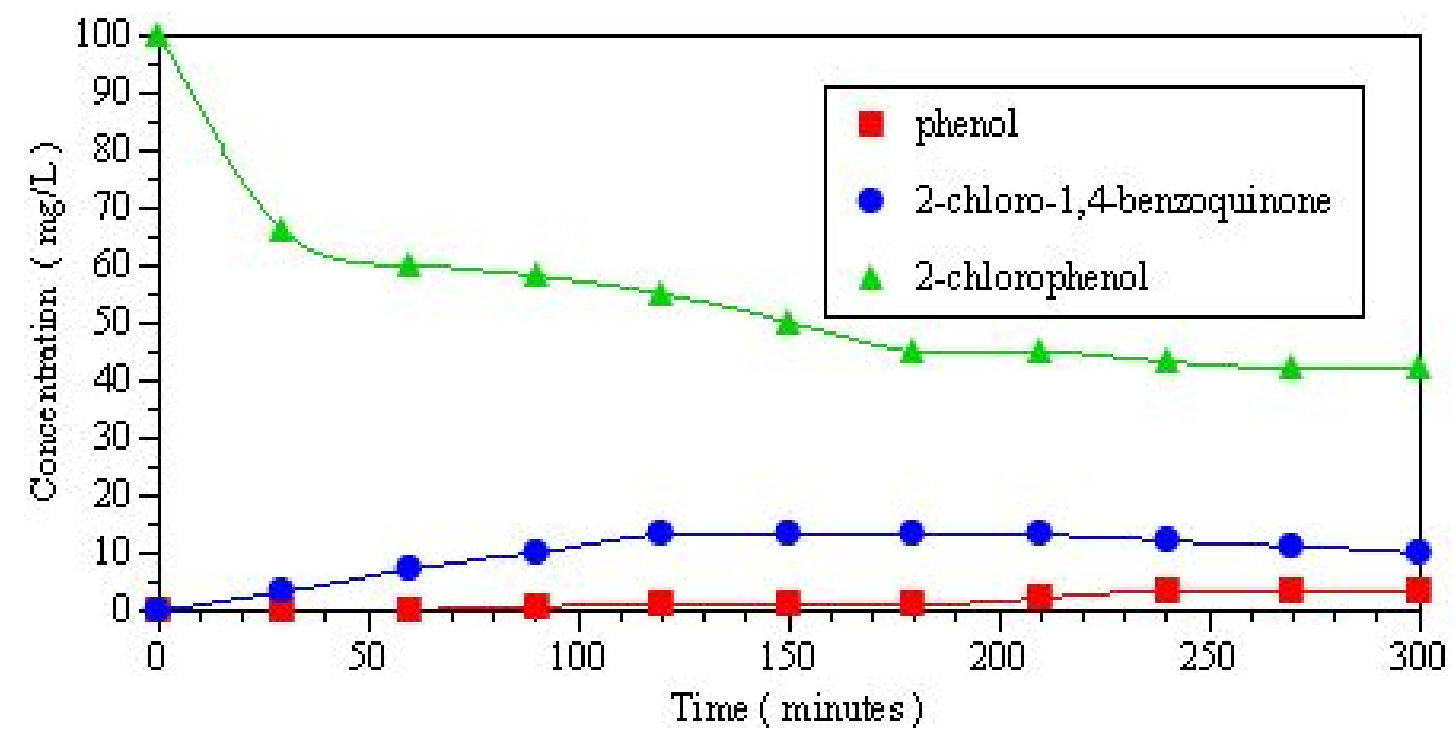

Figure 3. Reaction of 2-chlorophenol (1 mol) with potassium nitrosodisulfonate (4 moles) at room temperature and $\mathrm{pH} 5.5$.

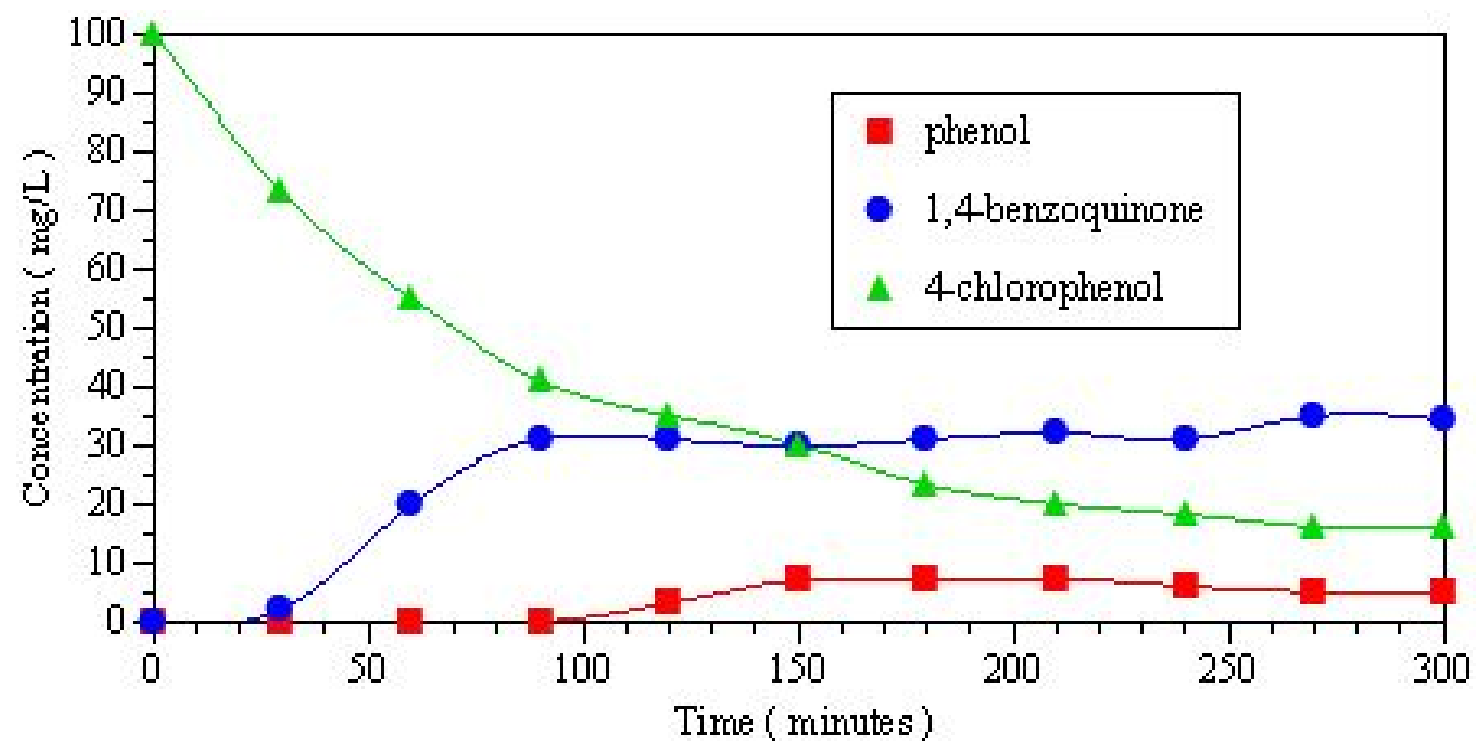

Figure 4. Reaction of 4-chlorophenol (1 mol) with potassium nitrosodisulfonate (4 moles) at room temperature and $\mathrm{pH}$ 5.3.

The concentration of 4-chlorophenol and its reaction products as a function of time is presented in Figure 4. In this case, 1,4-benzoquinone is formed in moderate yield. In addition, phenol is also formed in low yields. This reaction is particularly interesting since this procedure could be used for the partial oxidation of 4-chlorophenol to 1,4-benzoquinone.

In the case of 2,4-dichlorophenol, several intermediate products such as phenol, 2chlorophenol, 4-chlorophenol, and 2-chloro-1,4-benzoquinone were obtained (Figure 5). It is 
interesting to realize that at short reaction times, 2,4-dichlorophenol is almost quantitatively transformed into 4-chlorophenol that gives good yields at 90 minutes. After this time, 4chlorophenol begins to degradate and eventually all the intermediate compounds degradate to water, carbon dioxide and chlorine ions.

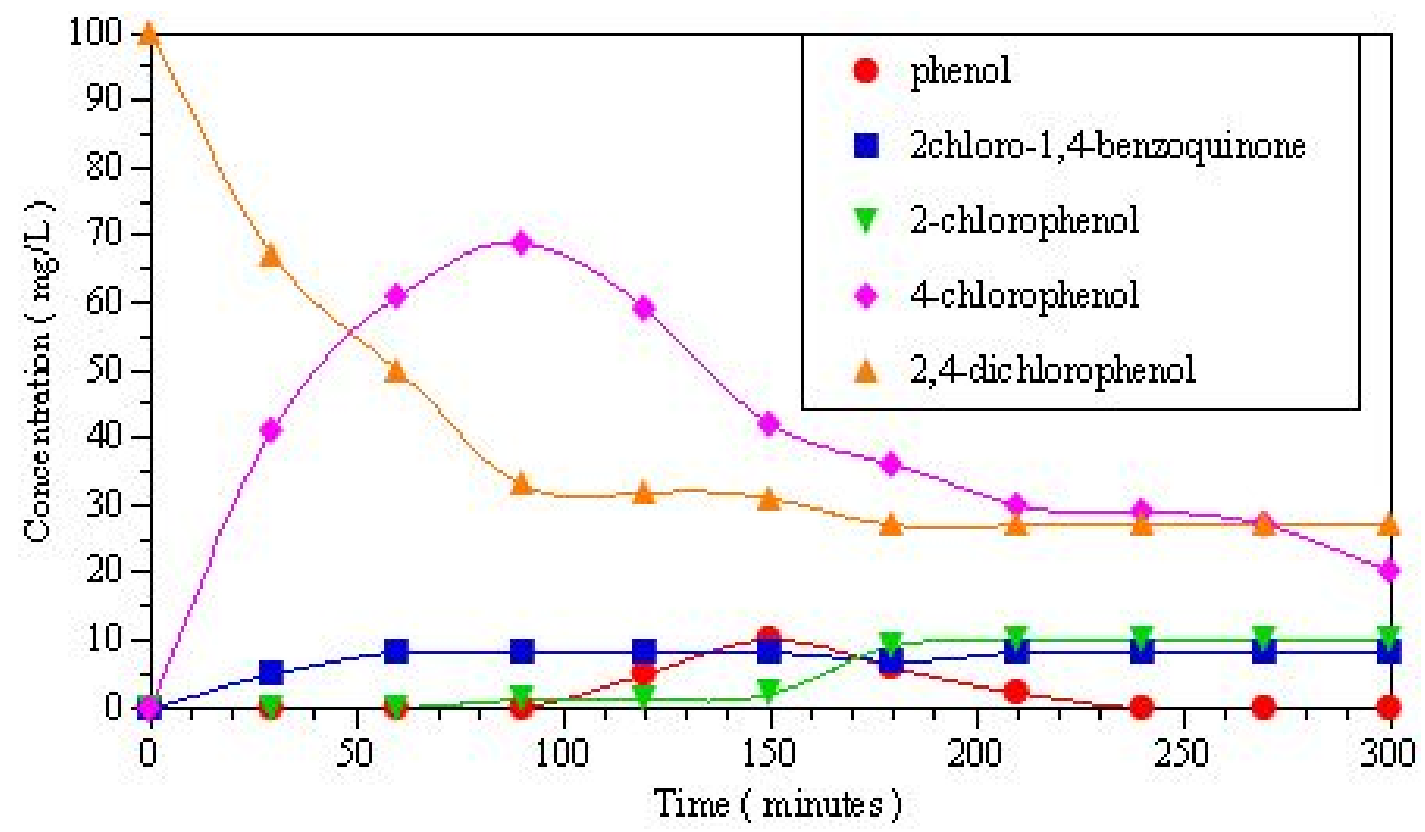

Figure 5. Reaction of 2,4-dichlorophenol (1 mol) with potassium nitrosodisulfonate (4 moles) at room temperature and $\mathrm{pH} 5.8$.

The chemical reaction of chlorophenols with potassium nitrosodisulfonate was monitored by ${ }^{1} \mathrm{H}$ NMR. In the case of 2-chlorophenol and 2,4-dichlorophenol, the corresponding aromatic signals ( 6 to $8 \mathrm{ppm}$ ) were observed to decrease as a function of reaction time. In the case of 4 chlorophenol, a strong band was observed to growth at $6.85 \mathrm{ppm}$ due to the presence of benzoquinone in the reaction mixture. Eventually, this and other bands were observed to disappear. The chemical reaction was also monitored by means of ${ }^{13} \mathrm{C}$ NMR. The signals due to aromatic carbon (110-114 ppm) were observed to decrease as a function of reaction time. After two hours of reaction, a signal at $182.6 \mathrm{ppm}$ due to $\mathrm{CO}_{2}$ was observed. This signal remained in the reaction mixture even at long oxidation times.

The mechanism of Fremy's radical oxidations has been extensively studied and has been fairly well established in the case of phenols. ${ }^{29}$ The overall stoichiometry of the oxidation of phenols has been shown to involve the reaction of 1 equivalent of phenol with 2 equivalents of potassium nitrosodisulfonate. The oxidation of hydroquinones also results in the formation of benzoquinones. A general mechanistic interpretation, consistent with the observed stoichiometry, has been suggested for Fremy's radical oxidation of phenols (Figure 6). 


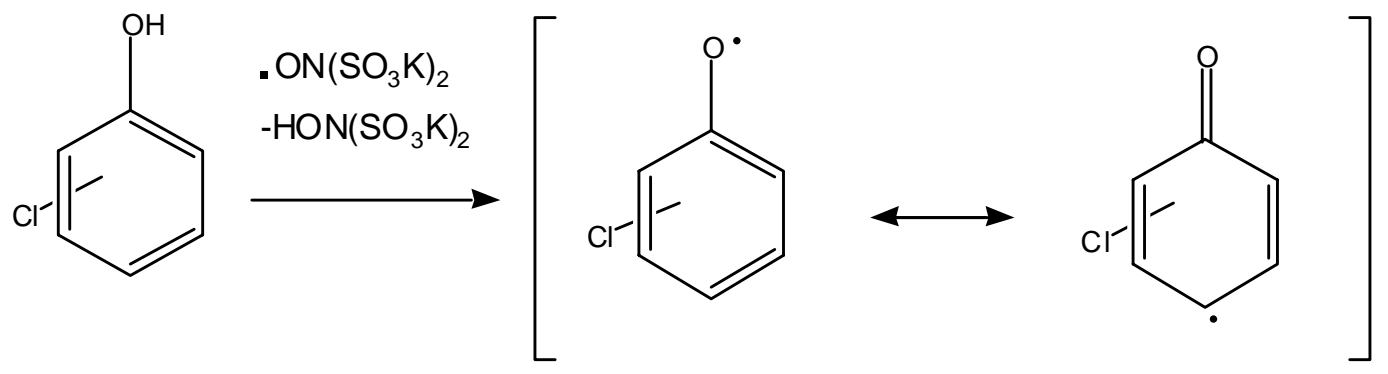<smiles>O=C1C=CC(=O)C(Cl)C1</smiles><smiles>CC1(C)C=CC(=O)C=C1</smiles>

Figure 6. Chlorophenol oxidation mechanism with potassium nitrosodisulfonate.

In summary, the selective chemical oxidation or reduction of several substituted chlorophenols (2-chloro, 4-chloro, and 2,4-dichloro) was carried out with potassium nitrosodisulfonate. The reaction was followed by NMR and HPLC. This reaction was strongly dependant on the amount of potassium nitrosodisulfonate, the position of the chloro-substituent on the aromatic ring, the $\mathrm{pH}$ and temperature of reaction. At short reaction times, 4-chorophenol was selectively oxidized to 1,4-benzoquinone and 2-chlorophenol to 2-chloro-1,4-benzoquinone. In contrast, 2,4-dichlorophenol was selectively reduced to 4-chlorophenol.

\section{Acknowledgements}

Financial support provided by Miguel Hidalgo-CONACyT through Grant CB06 is gratefully acknowledged. 


\section{References}

1. Keith, L. H.; Telliard, W. A. Environ. Sci. Technol. 1979, 13, 416.

2. Pellizetti, E.; Serpone, N. "Photodegradation of organic pollutants in aquatic systems catalyzed by semiconductors”, In Photocatalysis and Environment; Schiavello, M. Ed.; Kluwer Academic Publishers: Boston, 1988, p 469.

3. D’Oliveira, J. C.; Al-Sayed, G.; Pichat, P. Environ. Sci. Technol. 1990, 24, 990.

4. Besner, A.; Gilbert, R.; Tétreault, P.; Lépine, L.; Archambault, J. F. Anal. Chem. 1995, 67, 442.

5. Nilsson, C-A.; Norstrom, A.; Anderson, K.; Rappe, C. "Impurities in Commercial Products related to Pentachlorophenol”, In Pentachlorophenol. Chemistry, Pharmacology and Environmental Toxicology; Kao, R. R. Ed.; Plenum Press: New York, 1978, p 313.

6. Haggblom, M. Water Res. 1988, 22, 171.

7. Krijgsheld, K. R.; Van der Gen, A. Chemosphere 1986, 15, 825.

8. Boule, P.; Guyon, C.; Lemaire, J. Chemosphere 1982, 11, 1179.

9. Coats, J. R. CHEMTECH 1993, 25.

10. Pichat, P.; Mallard, C.; Guillard, C.; Courbon, H. Environ. Sci. Technol. 1994, 28, 2176.

11. Hasegawa, K.; Takeuchi, S. Environ. Sci. Technol. 1993, 27, 1819.

12. Pramauro, E.; Vincent, M.; Augugliaro, V.; Palmisano, L. Environ Sci. Technol. 1993, 27, 1790.

13. Vinodgopal, K.; Kamat, P. V. Environ. Sci. Technol. 1994, 29, 841.

14. Stafford, U.; Gray, K. A.; Kamat, P. V. J. Phys. Chem. 1994, 98, 6343.

15. Bolton, J. R.; Sun, L. J. Phys. Chem. 1996, 100, 4127.

16. Hoffman, M. R.; Martin, S. T.; Choi, W.; Bahnemann, D. W. Chem. Rev. 1995, 95, 69.

17. Leyva, E.; Moctezuma, E.; Ruiz, M. G.; Torres-Martínez, L. Catalysis Today 1998, 40, 367.

18. Tseng, J. M.; Hung, C. P.; Water Sci. Tech. 1991, 23, 377.

19. Vinodgopal, K.; Stafford, U.; Gray, K. A.; Kamat P.V. J. Phys. Chem. 1994, 98, 6797.

20. Stafford, U.; Gray, K. A.; Kamat, P. V. J. Catal. 1997, 25, 167.

21. Okamoto, K.; Yamamoto, Y.; Tanaka, H.; Tanaka, M. Bull. Chem. Soc. Jpn. 1985, 58, 2015.

22. Okamoto, K.; Yamamoto, Y.; Tanaka, H. Itaya, A. Bull Chem. Soc. Jpn. 1985, 58, 2023.

23. Jenks, W. S.; Li, X.; Cubbage, J. W.; Tetzlaff, T. A. J. Org. Chem. 1999, 64, 8509.

24. Jenks, W. S.; Li, X.; Cubbage, J. W. J. Org. Chem. 1999, 64, 8525.

25. Chiron, S.; Fernandez-Alba, A.; Rodriguez, A.; Garcia-Calvo, E. Wat. Res. 2000, 34, 366.

26. Bowers, A. R.; Gaddipati, P.; Eckenfelder, W.; Monsen, R. M. Wat. Sci. Tech. 1989, 21, 477.

27. Barbeni, M.; Minero, C.; Pelizzeti, E.; Borgarello, E.; Serpone, N. Chemosphere 1987, 16, 2225.

28. Hoigne, J.; Bader, H. Wat. Res. 1976, 10, 377.

29. Zimmer, H.; Lankin, D. C.; Horgan, S. W. Chem. Rev. 1971, 71, 229.

30. Leyva, S.; Crispin, I. Información Tecnológica 1998, 9, 317.

31. Pichat, P.; D’Oliveira, J.; Al-Sayyed, G. Environ. Sci. Technol. 1990, 24, 990. 